RESUMEN: El autor argumenta que resulta poco claro el papel que cumpla nuestra noción clásica de subjetividad en cualquier empresa cognitiva. En un segundo momento, trata de delinear un concepto útil de subjetividad que no se oponga binariamente al concepto de objetividad; $y$, hacia el final, traslada algunas de las conclusiones previas a sus consecuencias en el quehacer de las ciencias sociales.

PALABRAS ClAVE: subjetividad; privacidad; interioridad; Davidson; Wittgenstein.
ABSTRACT: The author states that the role of our classic notion of subjectivity is not clear in cognitive tasks. Secondly, he attempts to highlight the usefulness of a concept of subjectivity that has no binary opposition to objectivity. Finally, he will apply some of these previous arguments to his work in social sciences.

KEYWORDS: subjectivity, privacy, inner self, Davidson, Wittgenstein.

* Departamento de Filosofía, Universidad Autónoma de Aguascalientes. 


\section{¿ES LO SUBJETIVO UN MITO?}

$\mathrm{E}_{\mathrm{n} \text { su famoso artículo de } 1988, E l}$ mito de lo subjetivo, Donald Davidson cuestionó de manera rotunda la forma en la que hemos llegado a concebir la relación entre lo subjetivo y lo objetivo, entre la mente humana y la naturaleza. Este pretendido dualismo subjetivo/objetivo, y una serie de ideas asociadas a él — pensaba Davidson-debían ser rectificadas. Haciendo eco de algunas ideas de Davidson y Wittgenstein (entre otros), trataré de hacer dos cosas: en primer lugar, cuestionaré la inteligibilidad misma de nuestra concepción - heredada de la modernidad y el cartesianismo- de lo subjetivo. Para ello, pondré en duda tanto su aspecto semántico como su aspecto epistémico: cuestionaré la idea misma de un lenguaje privado, ${ }^{1}$ así como la idea de que el conocimiento subjetivo (el conocimiento de nuestra propia mente) es de un carácter especial y privilegiado. Hecho esto, queda poco o nada de nuestra vieja concepción de lo subjetivo. En un segundo momento, atiendo a algunas consecuencias metodológicas del dualismo subjetivo/objetivo, en particular a aquellas que nos han encaminado a dualismos no menos problemáticos: aquel entre ciencias de la naturaleza y ciencias del espíritu, entre metodologías cuantitativas y metodologías cualitativas, y entre ciencias naturales, y sociales y humanidades. Sin el viejo concepto de lo

${ }^{1}$ Aunque lenguaje privado y subjetividad no son la misma cosa, la posibilidad de un lenguaje privado es el correlato semántico de la subjetividad epistémica y metafísica. 
subjetivo, las dicotomías metodológicas deben ser abandonadas a favor de un monismo metodológico y un pluralismo explicativo. Para ello, defenderé una visión austera de naturalismo metodológico, así como una visión pluralista de los tipos de explicaciones, siempre en dependencia de nuestros intereses epistémicos.

Mi objetivo no es defender estas tesis por separado, algo que excedería los límites de este trabajo y requeriría defensas independientes. Me interesa, más bien, mostrar cómo todas estas posiciones pueden convivir coherentemente unas con otras. En otras palabras, que nada hay de paradójico o contradictorio en sostenerlas todas a la vez. Ahora vayamos por pasos.

\section{I}

Primero que nada, no sobra caracterizar brevemente el concepto cartesiano de lo subjetivo, el cual hemos heredado y permea innumerables de nuestras prácticas cognitivas. Según la concepción heredada, la oposición entre lo subjetivo y lo objetivo se aloja en la diferencia entre dos tipos de entidades: la naturaleza y la mente humana. Desde este punto de vista, existe una diferencia real entre la mente y el mundo. Conocemos la historia: mientras que el mundo es ma- terial, espacio-temporal, y se rige por leyes mecanicistas, la mente es inmaterial, solo temporal y se rige por leyes misteriosas. Las consecuencias de este dualismo ontológico han sido estudiadas hasta la saciedad: de este dualismo deviene nuestra idea de interioridad, privacidad, así como de subjetividad. Mientras que el mundo es público y externo, y puede ser estudiado de manera objetiva y cuantitativa, la mente es interna y privada, cognoscible solo de manera subjetiva y cualitativa.

Hay al menos dos reacciones distintas ante esta concepción dualista: la primera es el rechazo del estudio de lo subjetivo, relegando cualquier empresa cognitiva sobre lo subjetivo - en el mejor de los casos-a las ciencias blandas; la segunda - definida por el cartesianismo - opta por considerar a lo subjetivo como la piedra angular de todo nuestro conocimiento: nada más seguro — piensan ellos-, que el conocimiento de nuestra propia mente.

A partir del dualismo ontológico entre la mente y el mundo, podemos ver que se siguen una serie de dualismos de otra índole:

a) Dualismo semántico: entre el lenguaje psicológico (o mentalista) y el lenguaje físico descriptivo: los significados del primero vendrían dados 
por el sujeto que asigna significados a las palabras de dicho lenguaje a partir de su propio caso, generando innumerables lenguajes privados (tantos como sujetos); mientras que los significados del segundo serían intersubjetivos y, por tanto, públicos. b) Dualismo epistémico: entre el conocimiento que podemos obtener de nuestro estudio del mundo y el conocimiento de nuestra propia mente. c) Dualismo metodológico: entre las herramientas (metodologías) que usamos para conocer el mundo y las que usamos para conocer nuestra propia mente.

La tradición ha optado la mayoría de las veces por concentrarse en la validez o invalidez del dualismo ontológico. Los filósofos de la mente, de la psicología, los científicos cognitivos, incluso los neurocientíficos, han debatido con algidez los argumentos a favor y en contra del dualismo entre la mente y el mundo. Los pocos defensores hoy en día del dualismo ontológico han refinado sus argumentos, sabiendo que el dualismo cartesiano ha perdido toda su fuerza, y se han refugiado en posiciones mucho más sofisticadas, tales como el dualismo de propiedades, la teoría del doble aspecto y algunas formas de emergentismo. La amplia mayoría, por el contrario, ha optado por diversas formas de monismo fisi- calista. Sin embargo, el debate está lejos de poder cerrarse.

Por lo anterior, mi estrategia es inversa. No diré nada con respecto al dualismo ontológico. Cuestionaré tanto el dualismo semántico como el epistémico y el metodológico. Desde el punto de vista que adopto, si no tenemos buenas razones para defender estos dualismos, no tendremos tampoco ninguna buena razón para defender el dualismo ontológico. Empezaré entonces por cuestionar la inteligibilidad misma del concepto de lo subjetivo en su vertiente semántica y epistémica.

Si recordamos la caracterización anterior del concepto moderno de lo subjetivo, este parece tener algunas consecuencias semánticas importantes. Si la mente y el mundo son ontológicamente distintos - decíamoseso parece implicar que nuestras formas de hablar de ambos, de conocerlos y de estudiarlos deben ser distintas también. Estas formas distintas de hablar de ambos se hacen patentes en dos tipos de lenguajes: un lenguaje psicológico o mentalista que incluye términos como creencia, deseo, expectativa, emoción, sensación, etc., y otro lenguaje físico descriptivo, con el que nos referimos al mundo externo a la mente. Los significados del lenguaje mentalista dependerían del hablante: este le asignaría significados a los términos a 
partir de su propio caso: "dolor" significaría para Juan "el dolor que siente Juan". Así, habría inconmensurabilidad semántica entre el leguaje mentalista de Juan y el de Pedro, pues para ambos el significado de los términos mentales dependería de su propia experiencia interna, privada y subjetiva. Es este el origen de lo que se ha denominado como lenguaje privado: "Las palabras de este lenguaje deben referirse a lo que solo puede ser conocido por el hablante, a sus sensaciones inmediatas, privadas. Otro no puede, por tanto, entender este lenguaje". ${ }^{2}$ Esta concepción de un lenguaje privado fue expresada y defendida por Bertrand Russell en $L a$ filosofia del atomismo lógico:

Al hacer uso una persona de una palabra no le atribuye idéntico significado que cuando otra se sirve de la misma. He oído decir a menudo que esto último supone una contrariedad. Semejante opinión es infundada. Sería funesto que las personas significasen las mismas cosas mediante las palabras que usan. Ello haría imposible toda comunicación y convertiría al lenguaje en la menos prometedora y más inútil de las cosas imaginables, ya que el significado que ustedes confieran a sus palabras habrá de depender de la naturaleza de los objetos que conozcan direc-

${ }^{2}$ Ludwig Wittgenstein, Investigaciones filosóficas, 1988, México, UNAM, §243. tamente y, puesto que personas diferentes conocen directamente objetos asimismo diferentes, estas últimas no podrían dialogar entre sí a menos de que atribuyeran significados diferentes a sus palabras. De lo contrario, nos veríamos constreñidos a hablar de lógica - un resultado no del todo indeseable [...] Sería incalculablemente enojoso tener que servirnos de un lenguaje libre de ambigüedades y podemos, por tanto, congratularnos de no contar con él. ${ }^{3}$

La concepción russelliana de un lenguaje privado y el argumento que esgrime para defenderla son sumamente atractivos. Russell parece pensar que el hecho de no contar con significados compartidos hace útil la comunicación. Sin embargo, como veremos, la torna imposible. Wittgenstein esgrimió un argumento - conocido como el argumento contra el lenguaje privado - sumamente discutido en la segunda mitad del siglo XX, y que echa por tierra la posibilidad misma de la existencia de un lenguaje privado.

En primer lugar, habría que decir algo con respecto a lo que hace a este lenguaje un lenguaje privado. La privacidad de un lenguaje tal radicaría en los objetos a los cuales refiere. Las palabras de este lenguaje serían incomprensibles para cualquiera que

${ }^{3}$ Bertrand Russell, The philosophy of logical atomism, 1918, Abingdon, Routledge, pp. 22-23. 
no conociera directamente los objetos a los que dichas palabras hacen referencia; y, dado que las sensaciones de una persona A no las puede experimentar una persona $\mathrm{B}$ en lugar suyo, se concluye que B estaría incapacitado para comprender el significado de las palabras en cuestión o el lenguaje constituido por palabras con dichas características. Así, no habría posibilidad de que $\mathrm{B}$ comprendiese el lenguaje de $\mathrm{A}$, así como A no podría comprender el lenguaje de B. La posibilidad de un lenguaje privado, como el lenguaje de las sensaciones, socava cualquier pretensión de comunicación. Cualquier comunicación sería aparente - contra Russell: un sujeto A hablaría, en una situación ideal, con las mismas palabras que B, pero los significados serían distintos, pues las palabras harían referencia a objetos distintos, incognoscibles por cualquiera que no experimentase la sensación de manera directa. Son dichos objetos privados, las sensaciones de Ay B, los que imposibilitarían la mutua comprensión.

Contra la posibilidad de un lenguaje tal es contra la que Wittgenstein esgrime su argumento, el cual suele situarse entre los parágrafos 243 y 351 de las Investigaciones filosóficas. Delinearé brevemente el argumento, pero primero, recordemos: de acuerdo con la concepción dualista (cartesiana), los estados mentales son privados en el sentido específico de que solo son accesibles y cognoscibles de manera directa desde la perspectiva de la primera persona (de aquel que los tiene). Esto implica que están separados o solo tienen una relación contingente con el mundo público: es decir, un mundo en el que sus contenidos son igualmente accesibles y cognoscibles para más de una persona. Es en este sentido en el que, para la concepción cartesiana, los estados mentales son entidades privadas u objetos privados.

Ahora demos el siguiente paso. ¿A qué nos referimos con "estados mentales"? Para Wittgenstein, mientras elabora el argumento contra el lenguaje privado, su paradigma de estados mentales serán las sensaciones: aquellas que siento y a las que tengo un acceso privilegiado. En este sentido — como hemos visto-, la mente sería aquella cosa (teatro, receptáculo) con respecto a la cual solo yo tengo una perspectiva epistémica de primera persona. Así, mis estados mentales son transparentes: son aquello que yo creo que son.

Contra esta imagen, lo primero que hay que tener en cuenta es el giro wittgensteiniano: no hay acceso epistémico privilegiado, pues ni siquiera puede decirse en sentido estricto que yo sé que tengo una sensación, pues que realmente lo supiera implicaría 
que es posible que me equivoque al respecto. ${ }^{4}$ En este sentido, se podría decir más bien que los otros saben que tengo una sensación, mientras que yo no lo puedo decir respecto a mí mismo. Lo que Wittgenstein parece querer sugerirnos es lo siguiente: que yo no requiero un criterio para saber si experimento una sensación como dolor. Lo anterior quiere decir que yo no requiero un criterio de identidad para mis propias sensaciones.

Para Wittgenstein, el problema es esencialmente gramatical: nuestro juego del lenguaje de las autoatribuciones de estados mentales es tal, que si alguien cometiera errores al autoatribuirse dolor, lo que esto indicaría solamente es que él no ha comprendido la palabra “dolor". Aquí el problema parece estar en la noción misma de sujeto: si alguien a quien le preguntáramos si tiene dolor me dijera: "No lo sé, déjame ver", y empezara a analizar su propia conducta, diríamos que no ha entendido la diferencia crucial entre la gramática de la primera y tercera personas - entre decir yo y decir él-y que quizá no entiende la noción misma de sujeto.

Resumamos el punto hasta el cual hemos llegado: en la concepción cartesiana de la mente, el privilegio que tenemos con respecto a nuestros estados mentales se explica por su naturaleza privada. En otras palabras,

\footnotetext{
${ }^{4}$ Wittgenstein, op. cit., §246.
}

es porque solo yo lo sé, que realmente yo lo sé. Pero supongamos que en verdad nuestros estados mentales fuesen privados: ¿realmente tendríamos un acceso privilegiado a ellos que nos garantizara una referencia en primera persona a los mismos? Es en este contexto donde se comprende el célebre ejemplo wittgensteiniano del escarabajo:

Si digo de mí mismo que yo sé solo por mi propio caso lo que significa la palabra "dolor" - ¿no tengo que decir eso también de los demás? ¿Y cómo puedo generalizar ese único caso tan irresponsablemente?

Bien, ¡uno cualquiera me dice que él sabe lo que es dolor solo por su propio caso! - Supongamos que cada uno tuviera una caja y dentro hubiera algo que llamamos "escarabajo". Nadie puede mirar en la caja de otro; y cada uno dice que él sabe lo que es un escarabajo solo por la vista de su escarabajo. - Aquí podría muy bien ser que cada uno tuviese una cosa distinta en su caja. Sí, se podría imaginar que una cosa así cambiase continuamente. - ¿Pero y si ahora la palabra "escarabajo" de estas personas tuviese un uso? - Entonces no sería el de la designación de una cosa. La cosa que hay en la caja no pertenece en absoluto al juego de lenguaje; ni siquiera como un algo: pues la caja podría incluso estar vacía. —No, se puede "cortar por lo 
sano" por la cosa que hay en la caja; se neutraliza, sea lo que fuere.

Es decir: si se construye la gramática de la expresión de la sensación según el modelo de "objeto y designación", entonces el objeto cae fuera de consideración por irrelevante. ${ }^{5}$

Aquí el concepto clave — con el cual trabajó Wittgenstein arduamente casi los primeros cien parágrafos de las Investigaciones - es el de juego del lenguaje. Para Wittgenstein, un juego del lenguaje es "el todo formado por el lenguaje y las acciones con las que está entretejido". ${ }^{6}$ También, Wittgenstein considera que un juego del lenguaje es una práctica, con reglas al menos implícitas, en la que más de una persona puede participar. Por último, señala que los juegos del lenguaje son de distintos tipos y que muchos problemas filosóficos surgen cuando tratamos de entender un juego a partir de las reglas de otro juego. Ahora bien, el núcleo del argumento esbozado en el parágrafo 293 es el siguiente: nosotros no podemos introducir - piensa Wittgenstein - en un lenguaje público un término que refiera (en ese lenguaje) a un objeto privado. Si tenemos en mente el caso del escarabajo, la situación sería la siguiente: si alguien usa la palabra "escarabajo" justo como yo la uso, incluso aunque

${ }^{5}$ Ibid., §293.

${ }^{6}$ Ibid., $§ 7$. no hubiese nada dentro de su caja, ambos acordaríamos que lo que él tiene es un escarabajo, y que "escarabajo" es la palabra adecuada para nombrar aquello que él tiene. Así, no podría ser un objeto privado al cual nos estemos refiriendo, por lo cual los objetos privados - nos dice Wittgenstein- quedan fuera de consideración por irrelevantes.

Sólo resta una última posibilidad. Alguien podría argumentar lo siguiente: a pesar de que no podamos hacer referencia a objetos privados en un lenguaje público, podríamos disponer de otro lenguaje para nuestro uso propio en el cual hiciéramos referencia a lo que solo podemos conocer desde la perspectiva epistémica de la primera persona. Y este lenguaje nadie podría entenderlo, dado que nadie podría saber si estoy usando las palabras correctamente. Contra esta segunda suposición, el argumento contra el lenguaje privado es mucho más sutil: es parte de la gramática de las palabras que refieren a sensaciones en nuestro lenguaje público — piensa Wittgenstein- el que no cometamos errores cuando las aplicamos a nosotros mismos, siempre y cuando comprendamos su significado. Quien usara este lenguaje privado además del público, estaría suponiendo que tiene las mismas garantías, en lo que respecta a su lenguaje privado, que tiene quien usa el lenguaje 
público de las sensaciones para hablar de sus propias sensaciones. Sin embargo, dicha garantía pertenece a la gramática del lenguaje público, por lo cual no puede suponer que dispone de la misma en su lenguaje privado. En otras palabras: ¿cómo sabría el usuario de este lenguaje privado que la palabra $S$, que usa para designar un objeto privado en este momento, designa el mismo objeto privado que designaba la última vez que usó la palabra? ¿Cómo sabría siquiera que $S$ designa un objeto privado? El punto es que estas preguntas no tienen respuesta desde la perspectiva del usuario de este lenguaje privado. Él nunca podrá saber si está equivocado ni tampoco sabrá si estaba en lo correcto. Tampoco puede apelar a otros usuarios del lenguaje, dado que dicho lenguaje solo él lo comprende. Por último, dado que no hay un criterio de corrección en el uso de este supuesto lenguaje, no hay forma de saber siquiera si este designa verdaderamente objetos privados. Así -concluirá Wittgenstein-, parece que la idea misma de lenguaje privado carece de sentido.

De este modo, si el argumento contra el lenguaje privado es válido, parece que nos dice algo como lo siguiente: dado que no podemos hacer referencia a objetos privados en un lenguaje público y tampoco podemos hacer referencia a ellos en un lenguaje privado, no podemos referirnos a ellos en absoluto. A partir de este razonamiento, la concepción cartesiana de la mente y lo subjetivo sufre un fuerte revés: si el argumento contra el lenguaje privado es válido y aceptamos a su vez la concepción cartesiana, esto implicaría que no podríamos hablar siquiera de la mente. En conclusión: todo aspecto subjetivo es irrelevante desde un punto de vista semántico: el lenguaje es esencialmente intersubjetivo y la subjetividad queda fuera de cualquier consideración.

Ahora pasemos al dualismo epistémico. Descartada la posibilidad de articular inteligiblemente una concepción tradicional de lo subjetivo desde un punto de vista semántico, queda la posibilidad que podamos articularla desde un punto de vista epistémico: que lo subjetivo se aloje en algo que podemos conocer solo desde la perspectiva (subjetiva) de la primera persona. La tradición poscartesiana ha pensado que el conocimiento de nuestra propia mente cumple las condiciones anteriores: solo yo puedo conocer de una manera especial y privilegiada los contenidos de mi propia mente. En este sentido, lo subjetivo tendría lugar en el autoconocimiento. Las herramientas cartesianas para el autoconocimiento son la conciencia y la introspección: mediante ellas — pensaba 
el cartesianismo- podíamos obtener de manera transparente un conocimiento infalible y privilegiado de nuestros propios estados mentales.

Fue Gilbert Ryle quien, en $E l$ concepto de lo mental, cuestionó las supuestas garantías que nos otorgaban la conciencia y la introspección. ${ }^{7}$ Ryle argumentó que tanto la introspección como la conciencia constituían el mito del doble acceso privilegiado, y mostró por qué tenemos buenas razones para sospechar que somos constantemente falibles con respecto al conocimiento de nuestra propia mente, entre otras razones, Ryle señalaba que el autoconocimiento podía caer fácilmente en el problema judicativo de que el sujeto es juez y parte respecto al conocimiento de su propia mente; así como que hay estados mentales - en especial emociones, como la ira o la tristeza- que nos impiden conocer de manera objetiva los contenidos de nuestra propia mente (haciendo eco de un argumento brindado por Hume). ${ }^{8}$

Ahora bien, independientemente de que las supuestas herramientas de las que disponemos para el conocimiento subjetivo de nuestra propia

\footnotetext{
${ }^{7}$ Véase Gilbert Ryle, El concepto de lo mental, 1967, Buenos Aires, Paidós; especialmente el capítulo 6 .

${ }^{8}$ Esto lo he desarrollado con amplitud en otro lugar; véase Mario Gensollen, "Conductismo lógico y autoconocimiento, Alegato en pro de la primera persona", Tópicos 31, pp. 103-134.
}

mente sean desacreditadas, alguien podría sugerir que la interioridad y la privacidad de la propia mente siguen dando un espacio a lo subjetivo. Así resume esta concepción moderna de la subjetividad George Steiner:

Nadie ni nada puede, de manera verificable, penetrar mis pensamientos. Hacer que otro ser humano lea los pensamientos de uno no es más que una figura retórica. Puedo ocultar por completo mis pensamientos. Puedo disfrazar y falsificar su expresión externa lo mismo que puedo hacerlo con mi lenguaje gestual o corporal. Las plañideras contratadas claman de dolor sobre los restos de unos clientes a los que no conocen. $\mathrm{Ni}$ siquiera la tortura puede arrancarme más allá de toda duda mis pensamientos más íntimos. Ningún otro ser humano puede pensar mis pensamientos por mí. ${ }^{9}$

Sin embargo, dicha concepción es falsa. Ya sabemos que el lenguaje que utilizamos para referirnos a nuestros estados mentales es el mismo que usamos para referirnos a los estados mentales de otros. También, empíricamente, ahora sabemos que no hay nada en principio que me impida saber que el otro me engaña. El fantasma de la mentira y el fingimiento han sido desterrados para

${ }^{9}$ George Steiner, Diez (posibles) razones para la tristeza del pensamiento, 2007, México, FCESiruela, pp. 27-28. 
siempre de nuestra concepción de la intersubjetividad. Paul Ekman, ${ }^{10}$ siguiendo una intuición darwinista, ${ }^{11}$ ha demostrado que los gestos faciales $-\mathrm{y}$, en particular, las microexpresiones - contradicen nuestra verbalización y terminan siendo indicadores fiables de engaño. Wittgenstein, por su parte, en sus Últimos escritos de filosofía de la psicología, proporcionó una gramática del fingimiento que cuestionaba seriamente los conceptos de interioridad y privacidad antaño ligados a nuestra concepción de la mente. ${ }^{12}$

Ahora bien, si no tiene sentido articular el concepto de lo subjetivo ni desde una perspectiva semántica ni desde una epistémica, ¿qué nos queda de dicho concepto? Davidson concluye así El mito de lo subjetivo:

¿Qué queda entonces del concepto de subjetividad? En lo que se me alcanza, hay dos rasgos de lo subjetivo tal y como se conciben clásicamente que quedan en su sitio. Los pensamientos son privados en el obvio

${ }^{10}$ Véase Paul Ekman, "Why don't we catch liars?", Social Research 63-3; 801-17; también Paul Ekman, Telling lies: Clues to deceit in the marketplace, 1985, Nueva York, W. W. Norton.

${ }^{11}$ Charles Darwin, The expression of the emotions in man and animals, 1872, Nueva York, Oxford University Press.

${ }^{12} \mathrm{He}$ estudiado esta gramática del fingimiento con cierto detalle en otro lugar; Mario Gensollen, Seamos humanos, Wittgenstein y el giro romántico, 2009, México, Universidad Autónoma de Aguascalientes, pp. 95-122. pero importante sentido en que una propiedad puede ser privada, esto es, perteneciente a una persona. Y el conocimiento de los pensamientos es asimétrico en el sentido en que una persona que tiene un pensamiento por regla general sabe que lo tiene de una manera en que los otros no lo saben. Pero esto es todo lo que hay de subjetivo. ${ }^{13}$

Del viejo concepto de lo subjetivo, entonces, queda poco o nada. En efecto, soy yo el que tiene el pensamiento, la sensación o la emoción, pero no hay nada al respecto de dichos estados mentales de lo que no pueda expresarte con nuestro lenguaje compartido, ni nada con respecto a ellos que tú no puedas saber. Si esto es cierto, el concepto rectificado de lo subjetivo no se opone binariamente a lo objetivo. Es más, el conocimiento subjetivo sería objetivo en el obvio sentido de que su verdad es independiente de que sea crea que es verdad.

\section{II}

Llegados a este punto, el dualismo metodológico ha perdido toda su fuerza: si no podemos articular el viejo concepto de lo subjetivo ni semántica ni epistémicamente, no

${ }^{13}$ Davidson, "El mito de lo subjetivo", en Subjetivo, intersubjetivo, objetivo, 2003, Madrid, Cátedra, pp. 72-90. 
tiene sentido defender que requerimos dos metodologías distintas. Con la caída del concepto de lo subjetivo, las viejas fronteras entre las ciencias de la naturaleza y las ciencias del espíritu, entre las ciencias naturales y las ciencias sociales y las humanidades, entre metodologías cuantitativas y cualitativas, deben abolirse. A algunos, sin duda, este panorama puede parecerles desalentador, cuando no escandaloso. Sin embargo, con la caída de los viejos muros, los pasos que han empezado a dar las ciencias sociales y las humanidades son más sólidos que nunca.

Por ejemplo, Steven Pinker mostró con lujo de detalle, en sus conferencias Tanner de 1999, que la naturaleza humana — antes abandonada por las ciencias naturales - cada vez lograba incorporarse con mayor éxito a los estudios de cuatro nuevas ciencias: las ciencias cognitivas, las neurociencias, la genética del comportamiento y la psicología evolutiva.

Para los cognitivistas,

la vida mental podía explicarse en términos físicos a partir de los conceptos de información, computación y retroalimentación. Dicho llanamente: las creencias y los recuerdos no son otra cosa que información, la cual reside en ciertas estructuras y patrones de la actividad del cerebro. Pensar y planificar son secuencias de transformaciones de esos patrones. Querer e intentar son estados-meta que gobiernan esas transformaciones a través de un sistema de retroalimentación basado en las discrepancias halladas en el mundo entre el estadometa y la situación presente, y que las transformaciones tienen por objeto reducir. Esta idea general, que podríamos llamar teoría computacional de la mente, también explica como la inteligencia y la racionalidad pueden surgir de un mero proceso físico. ${ }^{14}$

En segundo lugar, la neurociencia trabaja con la que Francis Crick denominó "hipótesis asombrosa", a saber, que todos los aspectos del pensamiento y el sentimiento humano son manifestaciones de la actividad fisiológica del cerebro. En otras palabras, que la mente es lo que hace el cerebro, y en particular el procesamiento de la información que este lleva a cabo. Con respecto a la genética del comportamiento, su hipótesis también puede formularse de manera sencilla: todo el potencial para el aprendizaje y la experiencia compleja que distingue a los humanos de otros animales reside en el material genético que contiene el óvulo fertilizado. Por último, la psicología evolutiva (o el estudio de la historia filogenética y de las funciones adaptativas de la mente), aspira a "comprender el diseño de la

${ }^{14}$ Steven Pinker, La tabla rasa, el buen salvaje y el fantasma en la máquina, 1999, Barcelona, Paidós, p. 16. 
mente, no en ningún sentido místico o teleológico, sino como la omnipresencia del diseño o de la ilusión de diseño en el mundo natural (por ejemplo en el ojo o en el cerebro) y que Darwin explicó con la teoría de la selección natural". ${ }^{15}$

Para completar el panorama, otras ciencias, antes separadas de las ciencias naturales, ahora conviven también de cerca con la metodología naturalista. La antropología, por ejemplo, ha comenzado a estudiar la cultura de manera completamente natural; el trabajo de Dan Sperber ${ }^{16}$ es un ejemplo.

¿Qué queda al dualismo metodológico? La opción más evidente es el monismo metodológico. Dicho monismo, relegada la vieja concepción de lo subjetivo, debiera ser naturalista. Pero, ¿a qué me refiero exactamente con naturalismo? Empezaré con algunas ideas generales, tratando de delinear posteriormente sus tesis centrales.

Los naturalistas rechazan la postulación de entidades sobrenaturales para cualquier fin explicativo: divinidades, demonios, almas, fantasmas. La extensión sobrenatural está sujeta a amplia controversia en la explicación de cualquier ámbito de lo real. En segundo lugar, los naturalistas valoran

\footnotetext{
${ }^{15}$ Loc. cit.

${ }^{16}$ Véase Dan Sperber, Explaining culture. A naturalistic approach, 1996, Oxford, Blackwell.
}

tanto los resultados como los métodos de la ciencia; esto muchas veces les ha ocasionado el apelativo acrítico y peyorativo de "cientistas". En tercer lugar, los naturalistas introducen consideraciones empíricas en áreas antes ajenas a la introducción de este tipo de consideraciones (en especial en las ciencias sociales y las humanidades); por tanto, sospechan de cualquier consideración a priori en nuestras empresas cognitivas. Por último, los naturalistas defienden el falibilismo, es decir, reconocen que cualquiera de nuestras creencias puede ser, en principio, falsa; para ellos no hay verdades incontestables o fragmentos del saber humano que estén exentos de revisabilidad.

Dicho ahora de manera un poco más estricta, el monismo metodológico naturalista implica tres tesis:

(i) Que toda la evidencia de la que disponemos en nuestras empresas cognitivas debe ser, al menos en principio, públicamente observable.

(ii) Que los criterios de los que disponemos para evaluar las evidencias deben ser, al menos en principio, públicamente corregibles.

(iii) Y que todas las entidades que postulemos como existentes en nuestras explicaciones sean físicas.

Una consecuencia importante de la metodología naturalista implica que todos los conceptos no natu- 
rales que empleamos en la explicación del mundo deben ser:

(a) $\mathrm{O}$ reducidos o reconstruidos en términos de términos de tesis naturalistas respetables;

(b) $\mathrm{O}$ tratados de manera instrumental como ficciones útiles;

(c) O construidos sin un papel referencial o fáctico;

(d) $\mathrm{O}$ eliminados como manifestaciones de pensamiento precientífico.

Lo anterior indica un compromiso implícito del monismo metodológico naturalista con la tesis de la unidad de la ciencia.$^{17}$ El biólogo Edward Osbourne Wilson le he llamado a este proyecto "consiliencia", que significa literalmente que las ciencias se han unificado y cohesionado todas de un salto. Lo que esto significa es que las ciencias naturales han llegado a un estado de optimismo con respecto a la última de las fracturas dentro del paisaje del conocimiento humano: aquella entre biología o cultura, naturaleza o sociedad, materia o mente, ciencias o artes y humanidades. Dichas dicotomías hasta hace muy poco conservaban su respetabilidad a pesar de la caída de otros muros que dividían el conocimiento humano. Sin embargo, derrocada nuestra vieja imagen de

${ }^{17}$ Véase E. O. Wilson, Consilience. The unity of knowledge, 1996, Nueva York, Vintage. lo subjetivo, dichas dicotomías se desploman - como hemos vistopor sí solas.

Solo resta un último punto. Hasta aquí algunos científicos sociales y humanistas pueden sentir malestar. Pueden pensar que sus disciplinas han perdido toda su especificidad y que su función en la gran empresa del conocimiento humano no queda nada clara. La reacción común de los científicos sociales y humanistas frente al naturalismo es tacharlo de "cientista" o "reduccionista". Cabe decir algo al respecto, pues tales acusaciones suelen estar mal dirigidas, cuando no faltas de fundamento.

El naturalismo no implica necesariamente reduccionismo, si por reduccionismo entendemos que las explicaciones características de los científicos sociales y los humanistas deban ser abandonadas en pos de explicaciones de otra índole. El naturalismo puede y debe convivir con el pluralismo explicativo. La formulación más antigua y simple del pluralismo explicativo se la debemos a Aristóteles. En su Ética a Nicómaco, señala que

es propio del hombre instruido buscar la exactitud en cada género de conocimientos en la medida en la que la admite la naturaleza del asunto; evidentemente, tan absurdo sería aprobar a un matemático que 
empleara la persuasión como reclamar demostraciones a un retórico. ${ }^{18}$

El punto aristotélico consistía en señalar que la exactitud de nuestros razonamientos y nuestras explicaciones depende del objeto de estudio. Sin embargo, también depende de nuestros intereses epistémicos y prácticos. El objeto de estudio, en efecto, delimita algunas de nuestras pretensiones cognitivas: en algunos temas (la mayoría) debemos contentarnos con razonamientos plausibles. Pero, también, la fineza de nuestras explicaciones depende de lo que buscamos con ellas, de lo que nos interesa saber. Eso lo sabe el naturalista, que no pretende eliminar explicaciones psicológicas, sociales o económicas, pues sabe que algunas veces dichas explicaciones son las que en efecto responden a nuestros intereses epistémicos. Sabe también que distintas explicaciones, algunas mucho más finas y detalladas que otras - todas naturalistas - , pueden convivir en el mismo paisaje del conocimiento humano. ${ }^{19}$ Para muchos que defendemos un monismo metodológico naturalista, ni el reduccionismo

${ }^{18}$ Aristóteles, Ética a Nicómaco, 1996, Madrid, Centro de Estudios Políticos y Constitucionales, 1094b, pp. 24-30.

${ }^{19}$ Esta tesis ha sido defendida, en particular con respecto a las explicaciones en ciencias sociales, por Frank Jackson y Philip Pettit, "In defense of explanatory ecumenism", Economics and Philosophy, pp. 8. ni el eliminativismo son deseables: la opción más prometedora, quizá, sea el pluralismo, o el ecumenismo explicativo (como lo llaman Pettit y Jackson).

Gilbert Ryle intuyó que el monismo metodológico naturalista no excluía la posibilidad de este pluralismo o ecumenismo explicativo. En su quinta conferencia Tarner de 1953, titulada "El mundo de la ciencia y el mundo cotidiano", ${ }^{20}$ ofreció una profunda y sugerente analogía que ahora trataré de sintetizar. ${ }^{21}$

Pensemos en un muy buen administrador universitario, tan bueno, que está al tanto de todo lo que tiene que ver con la universidad. Por su escritorio pasan las facturas de jardinería, sabe cuánta basura se produce semanalmente, tiene un conocimiento exacto del número de profesores que imparten clases, sabe cuántos proyectos de investigación están en marcha, cuántos ejemplares hay en la biblioteca, cuántos alumnos ingresaron el último periodo, etc. Alguien podría decir que el administrador universitario tiene que ver con todo lo que pasa en la universidad. Y así es, en efecto. Sin embargo, aunque dicho administrador sabe cuántos profesores imparten clase, no tiene

${ }^{20}$ Gilbert Ryle, Dilemmas. The Tarner Lectures 1953, 1954, Cambridge University Press, Londres, pp. $68-81$.

${ }^{21}$ Adecuo la analogía de Ryle y le hago algunos cambios importantes. 
que ver con los criterios académicos que se toman en cuenta para su contratación. Del mismo modo, aunque sabe cuántos ejemplares hay en la biblioteca, no tiene nada que ver con si dichos ejemplares son los más adecuados o los más actualizados. Tampoco sabe si los alumnos que ingresaron lo hicieron con altos estándares académicos y mucho menos sabe cuáles son las expectativas académicas que se tienen con respecto a su egreso. Aunque el administrador tiene que ver con todo lo que pasa en la universidad, no tiene que ver con todas estas cosas en todos sus aspectos. Algunos aspectos que no le competen al administrador seguramente le competen a otras autoridades universitarias. Del mismo modo — piensa Ryle — aunque toda la realidad es en efecto física y la física nos provee las explicaciones más exactas, detalladas y finas de la realidad en su conjunto, al físico no le competen otros muchos aspectos de la misma realidad. Para ello, hay químicos, biólogos, así como psicólogos, sociólogos o antropólogos. Sus explicaciones - abrazando las tesis generales del naturalismo - no se oponen a las del físico, sino que abordan otros aspectos de la misma realidad. Así, el paisaje del conocimiento es uno solo, pero se enriquece con distintas explicaciones, unas mucho más finas y detalladas que otras. Si una explicación sociológica, por ejemplo, es adecuada, seguramente podríamos hacer una reducción interteórica de dicha explicación a otras: como psicológicas o biológicas. Sin embargo, no es esto lo que siempre nos interesa. La multiplicidad de explicaciones tiene su razón de ser en la amplia diversidad de nuestros intereses tanto epistémicos como prácticos.

Hasta aquí, parece quedar claro que es posible abrazar el monismo metodológico naturalista sin comprometernos con un reduccionismo explicativo. Si admitimos esta posibilidad, las consecuencias se nos presentan deseables. Las viejas barreras entre disciplinas y tipos de disciplinas deben derribarse. La constante preocupación de los científicos sociales y los humanistas porque los científicos naturales acaparan todos los recursos para sus investigaciones también debería desaparecer. Todo el conocimiento es natural, en el sentido en el que lo he defendido. Siendo el paisaje del conocimiento uno solo, a pesar de la inmensa pluralidad ecuménica de las explicaciones, la interdisciplinariedad es el futuro de la investigación naturalista. Como bien decía Gilbert Harman, las fronteras entre las diversas disciplinas y departamentos, termina siendo solo de interés para los administradores..$^{22}$

${ }^{22}$ Agradezco a Francisco Javier Ramírez Miranda su ayuda, sugerencias y comentarios. 